\title{
Effects of Sidewall Compression and Relaminarization in a Scramjet Inlet
}

\author{
Tue Nguyen, - Marek Behr, $\stackrel{ \pm}{-}$ and Birgit Reinartz \\ Center for Computational Engineering Science, RWTH Aachen University, \\ 52056 Aachen, Germany \\ and

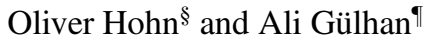 \\ DLR, German Aerospace Center, 51147 Cologne, Germany
}

DOI: $10.2514 / 1 . B 34740$

\begin{abstract}
This paper presents the numerical simulations and the performance analysis of a scramjet inlet as part of a combined experimental and numerical study. A well-validated finite volume flow solver was used to simulate a scramjet inlet with a double ramp configuration for outer compression, including varying degrees of sidewall compression. The computed wall pressure and heat transfer in the symmetry plane are in close agreement with the measurements, and the numerical results indicate that the weak sidewall compression alters the inlet performance significantly. The effects of partial relaminarization over the expansion corner, before the interior part of the inlet, is isolated and investigated in both the experiment and simulation. It is shown that relaminarization of a boundary layer is predicted accurately using the current numerical methods. This work represents a contribution to the understanding of the effects of sidewall compression and relaminarization in designing a scramjet inlet.
\end{abstract}

\section{Introduction}

$\mathbf{S}$ CRAMJETS (supersonic combustion ramjets) are hypersonic airbreathing propulsion systems using supersonic combustion. A scramjet inlet consists of several external compression ramps and an interior part. The flow is compressed through a series of oblique shock waves generated by the ramps and the sidewalls. The large adverse pressure gradient produced by the shock waves may cause the boundary layer to separate and results in a considerable increase in pressure losses and a blockage of the flow into the interior part. It is important in the early design phase to be able to quantify the performance of an inlet in terms of the compression ratio, the mass flow capture, and the flow conditions at the interface with the combustion chamber. Computational fluid dynamics (CFD) offers a practical and efficient means by which this requirement can be met.

This work is part of an ongoing research on scramjet engines in Germany [1]. A hypersonic inlet model was designed for the operating Mach number 6.5-7.5 with the upper limit chosen as the design point for shock-on-lip condition. The inlet was tested in the H2K tunnel at the German Aerospace Center in Cologne [2]. To consolidate the experimental database of scramjet inlets, a further study was performed to investigate the effects of sidewall compression [3] . Designing an inlet with sidewall compression is a

Presented as Paper 2011-2256 at the 17th AIAA International Space Planes and Hypersonic Systems and Technologies Conference, San Francisco, CA, 11-14 April 2011; received 15 July 2012; revision received 10 January 2013; accepted for publication 15 February 2013; published online 17 April 2013. Copyright (C 2013 by Tue Nguyen, Marek Behr, Birgit Reinartz, Oliver Hohn, and Ali Gülhan. Published by the American Institute of Aeronautics and Astronautics, Inc., with permission. Copies of this paper may be made for personal or internal use, on condition that the copier pay the $\$ 10.00$ per-copy fee to the Copyright Clearance Center, Inc., 222 Rosewood Drive, Danvers, MA 01923; include the code 1533-3876/13 and \$10.00 in correspondence with the CCC.

*Ph.D., Chair for Computational Analysis of Technical Systems; nguyen@ cats.rwth-aachen.de. Member AIAA.

${ }^{\dagger}$ Professor, Chair for Computational Analysis of Technical Systems.

${ }^{\ddagger}$ Research Fellow, Chair for Computational Analysis of Technical Systems. Senior Member AIAA.

${ }^{\S}$ Ph.D. Student, Supersonic and Hypersonic Technology Department, Institute of Aerodynamics and Flow Technology, Linder Höhe. Member AIAA.

"Head of Department, Supersonic and Hypersonic Technology Department, Institute of Aerodynamics and Flow Technology, Linder Höhe. Member AIAA. compromise between a longer inlet with a smaller compression angle, thus imposing a penalty on size and weight to obtain the required compression and a shorter inlet with a larger compression angle, which leads to stronger shocks from the sidewall and the increased probability of boundary-layer separation. Holland [4] showed that, when the location of the cowl is fixed, increasing the sidewall compression enhanced the total pressure recovery and the kinetic energy efficiency. However, the mass flow captured was reduced significantly. In this work, sidewall compression was achieved by introducing a smooth contraction along the outer part of the inlet. Because the measurements were only taken along the centerline of the inlet model, computational analysis is useful to obtain physical insights on the three-dimensional (3-D) flowfield. The numerical results are first validated by comparing against the experimental data, and then they are used to gain further understanding of the effects of sidewall compression.

Furthermore, the ability of common turbulence models to predict a rapid expansion in hypersonic flows has been analyzed. This normally takes place at the entrance of the interior part where the flow is being expanded along a convex corner or surface. Because turbulence is damped by the flow expansion and acceleration, and becomes less important to the development of the mean flow, the flow is normally considered as "relaminarizing" (though turbulence does not completely vanish). This phenomenon is also commonly referred to as "relaminarization," where the initially turbulent boundary layer returns to a laminar-like state in terms of velocity profile, skin friction, and heat transfer. In supersonic flows, relaminarization was observed in the accelerating flow around an expansion corner $[\underline{5}, 6]$. The criteria for the occurrence of relaminarization have been proposed by many authors [7]. For compressible turbulent flow over expansion corners, Narasimha and Viswanath [8] reviewed a wide selection of experiments and concluded that, below Mach 3, relaminarization would occur when $\Delta P / \tau_{0}$ is larger than 70 , where $\Delta P$ is the total pressure drop (considered as positive in an expansion) and $\tau_{0}$ is the upstream wall shear stress. This criterion is attractive because it takes the information from both the mean flow and the near-wall turbulence. Recent numerical simulations $[9,10]$ have shown that common turbulence models can replicate the mean flow features and the Reynolds stresses of relaminarizing flows reasonably accurately for Mach number up to three. In this paper, the validity of this statement and the relaminarization criterion in a higher Mach number regime will be verified by means of experiments and numerical simulations. 


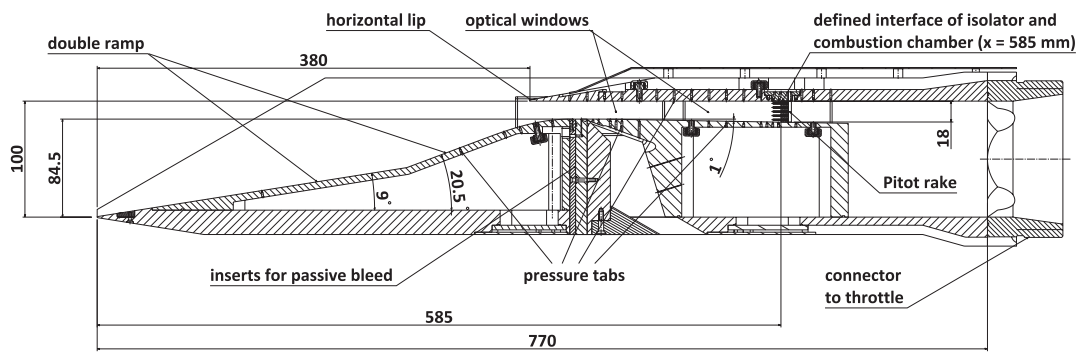

a)

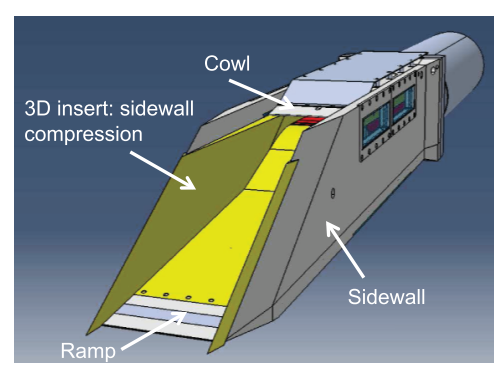

b)

Fig. 1 Schematic of a) CAD drawing of the symmetry plane of the inlet; b) inlet with mounted 3-D insert.

This paper is organized in the following way. Section II provides the information on the inlet model, the test condition, and the experimental techniques. Section III describes the numerical and the grid generation methods used in this context, including spatial discretization, time integration, boundary conditions, and turbulence modeling. The experimental and numerical results are then presented in Sec. IV, in which the effects of sidewall compression and relaminarization are discussed. Finally, conclusions are drawn in Sec. V.

\section{Experiment}

A schematic drawing of the scramjet inlet model used in this work is shown in Fig. 1a. The inlet is $585 \mathrm{~mm}$ long and $100 \mathrm{~mm}$ wide and comprises two external compression ramps and an interior part. The first and second ramps are inclined to the horizontal surface by 9 and $20.5 \mathrm{deg}$, respectively. The leading edges of the first ramp and the cowl lip are blunt, with a radius of $0.5 \mathrm{~mm}$. The second ramp and the interior part are connected by an isentropic expansion surface. The sidewalls have a constant sweep angle of $30 \mathrm{deg}$. Sidewall compression was achieved by mounting a 3-D insert [3] onto the original sidewall, as illustrated in Fig. 1b. This 3-D insert provides a smooth contraction in the outer part of the inlet and results in a reduced width of the interior part. Two different 3-D inserts were used: an 80 and a $70 \mathrm{~mm} 3-\mathrm{D}$ insert where the inserts are named after the resulting inlet width at the end of the sidewall compression. These reductions are equivalent to compression angles of approximately 1.7 and $2.5 \mathrm{deg}$ along the outer part of the inlet. In the relaminarization study, the 3-D insert was not used and the width remains constant at $100 \mathrm{~mm}$ from the leading edge of the inlet to the back of the interior part. Furthermore, the cowl was removed to isolate the effects of relaminarization.

The inlet was tested in the Hypersonic Wind Tunnel H2K at the DLR in Cologne [2,3]. This facility is a blowdown wind tunnel designed to simulate different Mach numbers and Reynolds numbers using contoured nozzles. Two test conditions were used in the experiments and are listed in Table $1 . M_{\infty}$ is the freestream Mach number, $R e_{\infty, m}$ is the unit Reynolds number based on the freestream condition, and $P_{0}$ and $T_{0}$ are the total pressure and the total temperature, respectively. Condition A was used for both studies of the effects of sidewall compression and relaminarization. Condition $\mathrm{B}$ was only used for the study of relaminarization to assess the effect of Reynolds number on this phenomenon. In the experiments, the pressure was measured by 42 static pressure ports along the centerline of the model, of which 25 are located on the ramps and the lower surface of the interior part. The Stanton number was deduced based on the data from the infrared thermography pictures [11]. The uncertainties of the pressure coefficients and the Stanton number are \pm 5.6 and $\pm 20 \%$, respectively [ 3 ]. The exact dimensions of the inlet,

Table 1 Test conditions in the experiments

\begin{tabular}{ccccc}
\hline \hline Condition & $M_{\infty}$ & $R e_{\infty, m}, 1 / \mathrm{M}$ & $P_{0}$, bar & $T_{0}, \mathrm{~K}$ \\
\hline $\mathrm{A}$ & 7.0 & $4 \times 10^{6}$ & 7 & 500 \\
$\mathrm{~B}$ & 7.0 & $10 \times 10^{6}$ & 28.5 & 630 \\
\hline \hline
\end{tabular}

the performance of the wind tunnel, and the detailed measurement techniques can be found in Hohn and Gülhan [3]

For clarity, the dimensionless pressure coefficient $C_{p}$ and Stanton number $S t$ are defined as follows:

$$
C_{p}=\frac{p-p_{\infty}}{0.5 \rho_{\infty} V_{\infty}^{2}}
$$

$$
S t=\frac{\dot{q}_{\text {conv }}}{\rho_{\infty} V_{\infty} C_{p, \text { air }}\left(T_{\text {rec }}-T_{w}\right)}
$$

in which $p$ is the local static pressure, $p_{\infty}$ is the freestream static pressure, and $\rho_{\infty}$ and $V_{\infty}$ are the freestream density and velocity, respectively. The convective heat flux is given by $\dot{q}_{\text {conv }}, C_{p \text {,air }}$ is the specific heat capacity of air at constant pressure, and $T_{\text {rec }}$ and $T_{w}$ are the recovery and wall temperatures, respectively. The recovery temperature is computed as

$$
T_{\mathrm{rec}}=\left(1+r \frac{\gamma-1}{2} M_{\infty}^{2}\right) T_{\infty}
$$

where $\gamma$ is the heat capacity ratio, $M_{\infty}$ and $T_{\infty}$ are the freestream Mach number and temperature, respectively, and $r=0.9$ is the recovery factor for turbulent flow.

\section{Computation}

\section{A. Numerical Method}

The computations were performed using a scientific research code QUADFLOW, which solves the three-dimensional Reynoldsaveraged Navier-Stokes (RANS) equations. QUADFLOW has been documented in Bramkamp et al. [12]. Here, only the most important characteristics with respect to the present study are mentioned. The spatial discretization of the governing equations is based on a cellcentered finite volume scheme. The flux splitting scheme from Wada and Liou [13] is used to discretize the convective fluxes, and secondorder accuracy is achieved by a linear reconstruction. The viscous fluxes are discretized by central differencing. The mean flow equations are integrated in time by an explicit five-stage RungeKutta scheme. QUADFLOW has been validated extensively with different test cases consisting of various flow conditions [12]. The flow solver has been proved to be suitable for supersonic and hypersonic computations [14].

Turbulence closure is achieved with the two-equation shear stress transport $k-\omega$ model [15]. Laminar-turbulent transition was found experimentally around the separation bubble at the kink between the first and second ramps [2]. This phenomenon is modeled by setting the production of the turbulent kinetic energy in all cells on the first ramp to zero, thus the flow is effectively laminar in this area. It will be shown later that this approach, which is named "transition box" here, provides reasonable agreement with respect to the experimental data.

The following boundary conditions are used in the computations: supersonic inflow, supersonic outflow, and solid wall. At supersonic inflow boundaries, the values are prescribed using the experimental data. The freestream turbulence intensity was fixed at $0.5 \%$, which is 


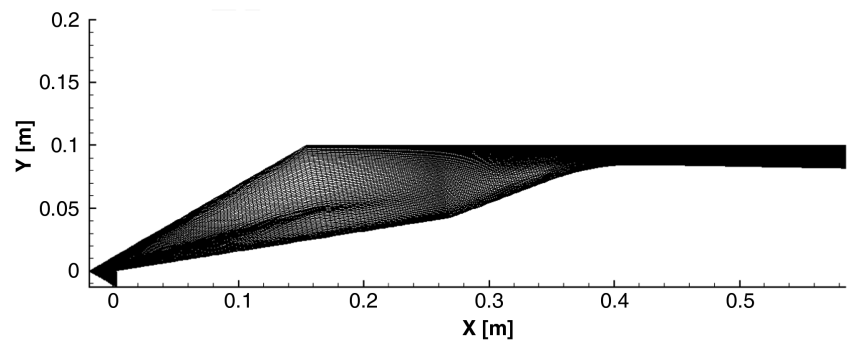

Fig. 2 Grid distribution in the symmetry plane of the inlet.

a good estimate for the $\mathrm{H} 2 \mathrm{~K}$ tunnel. At supersonic outflow boundaries, the variables are extrapolated from the interior, assuming zero gradient. At solid walls, the no-slip condition is enforced and the isothermal condition is used. Because the test time is relatively short (approximately 20 s), it is assumed that the wall temperature remains constant throughout the experiment $\left(T_{w}=300 \mathrm{~K}\right)$. The turbulent kinetic energy is set to zero at the wall and the respective length scale is prescribed based on the first grid spacing according to Menter [15].

\section{B. Grid Generation}

The grid used in this work was originally created by Krause [14] for the inlet without the sidewall compression using a multiblock elliptic structured grid generator (MegaCads [16]). The original grid was shown to be well constructed with high resolution and is able to provide flowfield that is in good agreement with the measurements [14]. In this work, the grid is further refined and modified for the geometries with the sidewall compression. The final grid represents half of the inlet and consists of 18 blocks. The total number of cells is close to 4 million with 608 grid points in the streamwise direction, 96 grid points in the wall normal direction, and 64 grid points in the spanwise direction.

Figure 2 illustrates the grid distribution in the symmetry plane of the inlet. It can be seen that the grid points in the wall normal direction are stretched by Poisson distribution to achieve $y^{+}$smaller than one close to the wall everywhere in the computational domain (the minimum distance to the wall is $10^{-6} \mathrm{~m}$ ). The transverse grid lines were created in such a way that they are almost always perpendicular to the wall surface. At the blunt leading edge, great care was taken to ensure that the grid lines approximately align with the bow shock and the grid resolution is sufficient to resolve the shock wave (see Fig. 3a). Because of the geometric complexity, the leading edge of the cowl lip is assumed to be sharp instead of blunt and the grid lines were clustered in this area to capture the lip shock (Fig. 3b). For the computations with the cowl removed, the grid is uniformly distributed along the upper boundary of the computational domain. Figure 4 shows the 3 -D grids of the inlet with and without sidewall compression, where the grid lines in the spanwise direction are also stretched toward the sidewall to resolve the boundary layer there.

\section{Results}

\section{A. Simulation of the Inlet Without Sidewall Compression}

In this subsection, the flow inside the inlet with only the original sidewall will be examined. Because the 3-D insert is not attached, there is no sidewall compression (SWC). The general flow features in

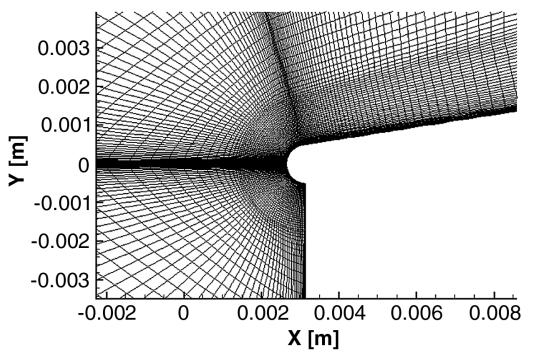

a)

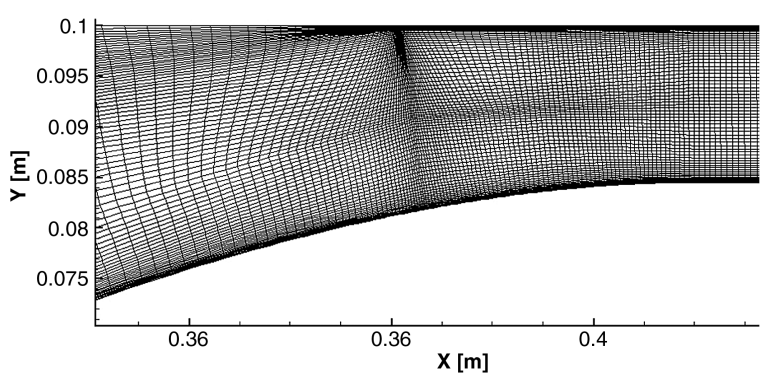

b)

Fig. 3 Grid distribution a) around the blunt leading edge of the first ramp and b) at the entrance of the interior part. Grid lines are stretched at the cowl lip $(x=0.38 \mathrm{~m})$.

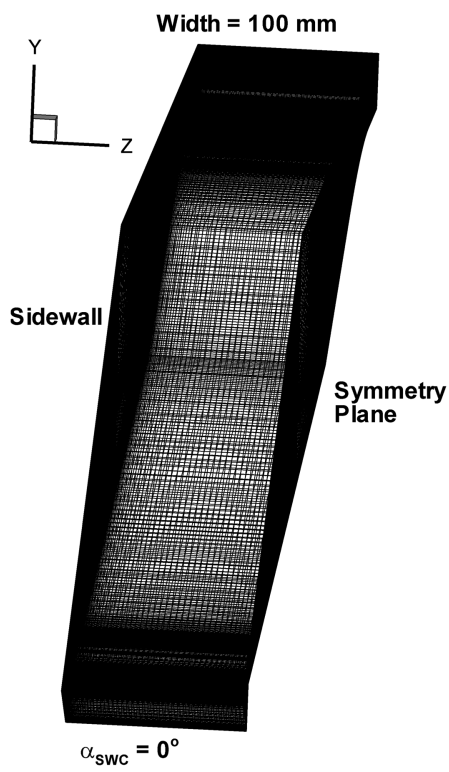

a)

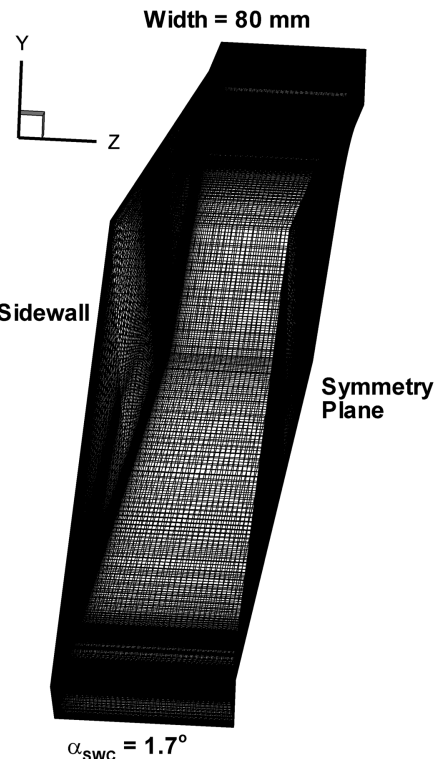

b)

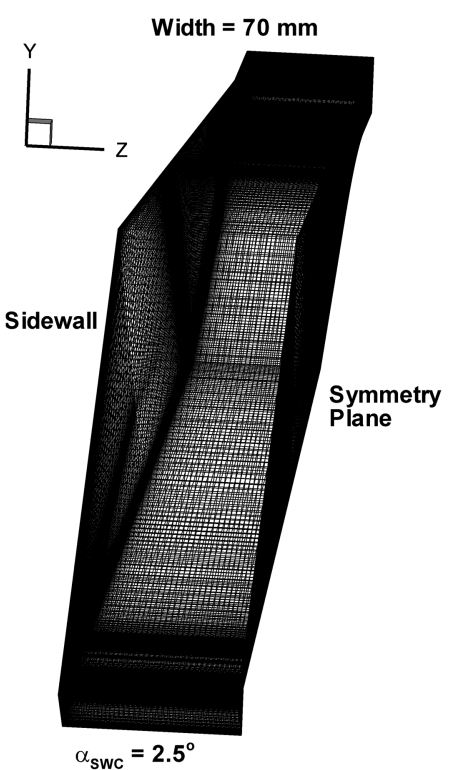

c)

Fig. 4 Grid distribution of the inlet with and without sidewall compression $\left(\alpha_{\text {SWC }}\right.$, sidewall compression angle). 

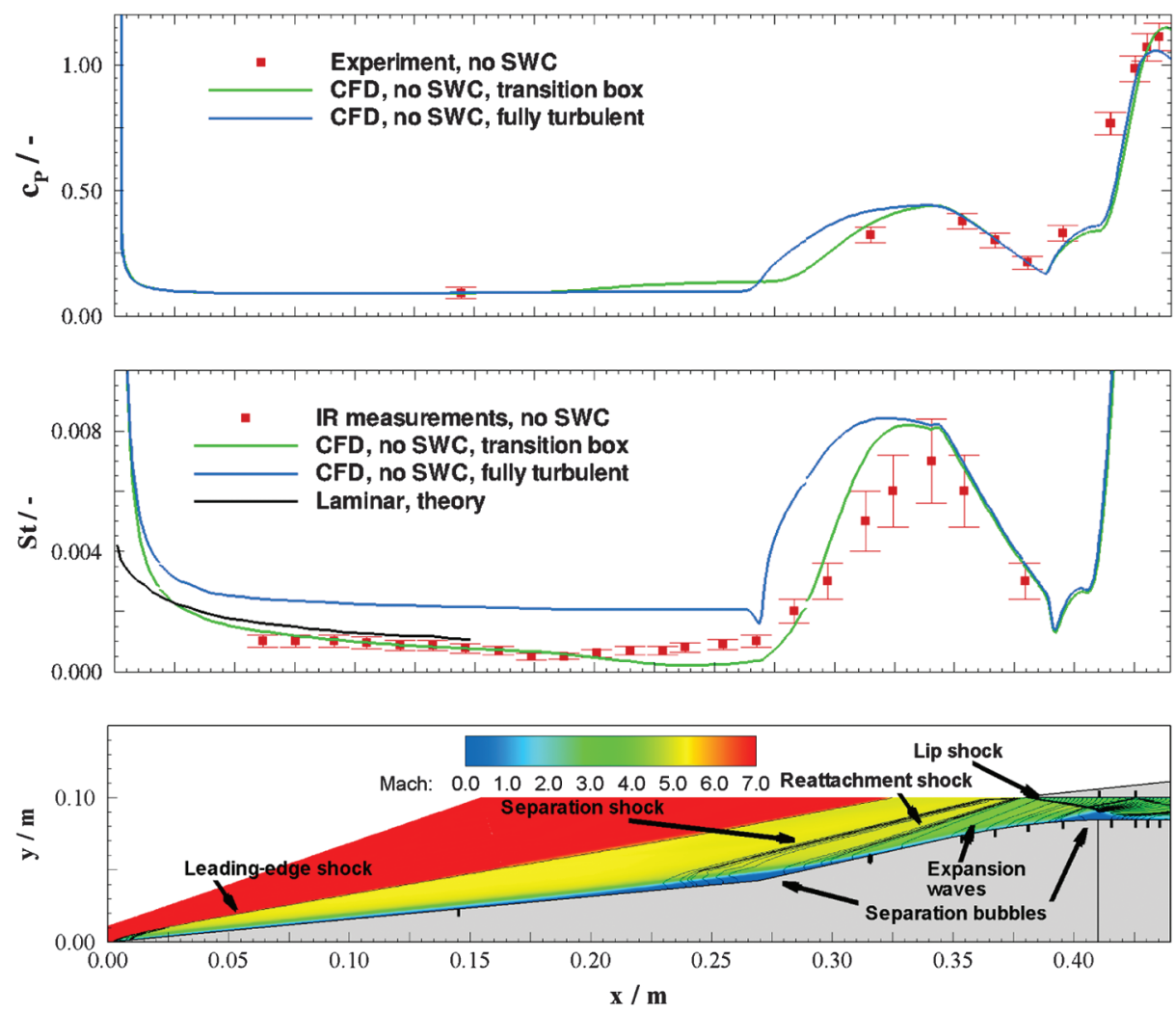

Fig. 5 Pressure and heat transfer along the bottom wall, in the symmetry plane of the inlet.

the symmetry plane of this configuration are illustrated in the bottom plot of Fig. 5. Here, the background is the Mach contours and the solid lines are the isopressure lines. The flow is first compressed by the bow shock from the blunt leading edge of the first ramp. The separation bubble between the first and second ramps is associated with a separation shock wave and a reattachment shock wave. At the entrance of the interior part, the flow experiences a supersonic expansion and relaminarization at the end of the last ramp before encountering an oblique shock wave from the cowl lip. The large adverse pressure gradient produced by this shock-boundary-layer interaction causes the boundary layer to separate from the inlet wall.

It was found in the experiment [2] that the flow was transitioning in the shear layer over the separation bubble between the first and second ramp. This phenomenon is modeled in the simulation by using a transition box, as described in the preceding section. The validity of this approach is shown in Fig. 5 , in which the wall pressure and the heat transfer distributions along the bottom wall of the symmetry plane of the inlet are compared with the measurements. For comparison purposes, a fully turbulent computation was also performed and included in this figure. Furthermore, the theoretical laminar heat transfer on the first ramp is computed using the formula given in Häberle and Gülhan [2]. Two distinct features are obvious in the computation with the transition box: The flow is laminar on the first ramp, which agrees much better with the experimental data and the theoretical values, and the adverse pressure gradient caused by the deflection on the second ramp induces a flow separation, which causes a small pressure plateau in the last part of the first ramp. In the fully turbulent computation, the boundary layer is less susceptible to adverse pressure gradients, thus there is no flow separation in between the ramps.

To understand the effects of the SWC, it is crucial to first understand the physics of the swept shock-boundary-layer interaction at the corner of the inlet. Here, because the sidewall shock is very weak in comparison with the ramp shock, the interaction can be classified as a fin-type configuration. Alvi and Settles [17] proposed a model of the flow structure for this type of interaction based on flow visualization. Along the flat plate, the fin shock acts as a swept shock wave that turns, compresses, and decelerates the near-wall flow. The degree of turning is not the same throughout the whole boundary layer because of the nonuniform Mach number. This causes the formation of a vortical motion that sweeps the high momentum fluid in the outer layer to the wall and removes the low momentum fluid from the wall [18]. The inviscid shock bifurcates into a separation shock and a rear shock, and a slip line emanates from the triple point to ensure that pressure and flow direction are the same downstream of the $\lambda$-shock structure. Furthermore, in between the slip line and the primary vortex, the flow is first accelerated through a series of expansion waves before becoming a high-speed impinging jet in the near-wall region.

Regarding the flow near the sidewall, all the main features in the model of Alvi and Settles [17] can be observed in the current computation, as illustrated in the Mach and total pressure contour plots in Fig. 6. This plot is taken from a plane normal to the freestream direction, at a certain distance downstream of the leading edge of the first ramp. The flow features are numbered as follows: 1) ramp shock, 2) separation shock, 3) rear shock, 4) slip line, 5) expansion region, 6) impinging jet, 7) primary vortex, 8) and 9) secondary vortices. The plot is oriented so that the view can be compared directly to the model from Alvi and Settles [17]. The $\lambda$ shock, the expansion region, and the primary vortex are visible in the Mach contour plot, whereas the slip line and the jet impingement are more clearly shown in the total pressure plot. At the wall, the impinging jet diverges underneath the primary vortex and the corner between the ramp and the sidewall and initiates two additional secondary vortices. These secondary vortices were observed in both experimental and numerical works by Kubota and Stollery [19] and Knight et al. [20].

Figure 7a shows the footprint of the flow structures on the ramps and the sidewall in terms of the Stanton number and the surface stream traces. The "Pt plot" refers to the total pressure ratio plot as illustrated in Fig. 6. On the first ramp, the separation bubble is visible in between the 1) line of separation and 2) the line of reattachment. It can be seen that the separation is highly three-dimensional, having a larger extent near the symmetry plane. A region of low Stanton number downstream of the separation line is followed by a region of increasing heat transfer around and downstream of the reattachment line, where the streamlines are compressed toward the surface. This 


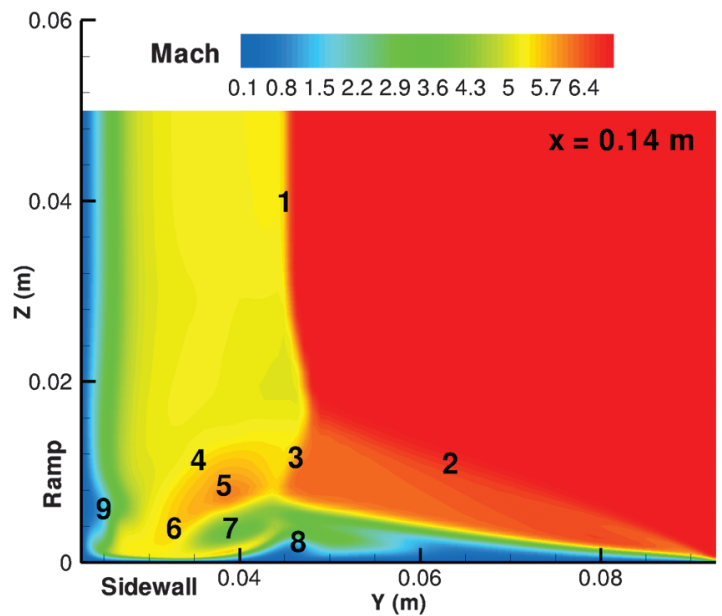

a) CFD, Mach number

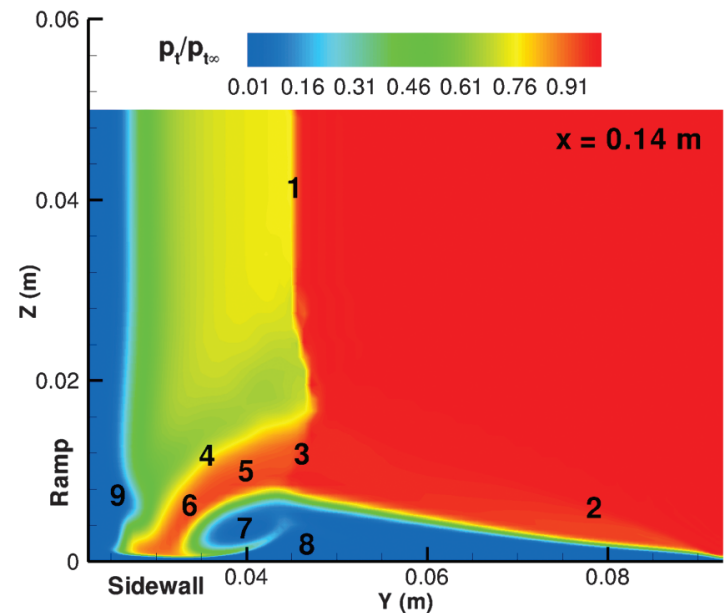

b) CFD, total pressure

Fig. 6 The flow structures near the sidewall in the scramjet inlet without SWC.

region is then interrupted at the onset of the isentropic expansion surface where the flow partly relaminarizes, visible by a decrease in the Stanton number (region 3). The flow is quite two-dimensional in the relaminarization region, except in the area close to the sidewall. At the corner between the ramps and the sidewall, the flow is heated in the vicinity of the corner due to the divergence of the jet impingement. Next to it, the presence of the corner (secondary) vortex leaves a trace of a low-heat transfer area (region 7). The surface structure on the ramps is in qualitative and quantitative agreement with the infrared picture taken during the experiment (see Fig. 7b), except for the very high-heat transfer region at the corner on the second ramp. The absence of this region in the infrared picture is probably due to the selected scaling, as explained by Häberle and Gülhan [2].

Along the sidewall surface, the footprints of the sidewall separation and the jet impingement are visible through a region of low Stanton number (around the sidewall separation, line 4) and a region of high Stanton number (region 6), respectively. The sidewall separation denotes the beginning of the formation of the primary vortex, whereas the jet impingement defines a region beyond which the flow is approximately parallel to the ramp [21]. In addition, the trace of the secondary vortex can also be seen as a low-heat transfer region in between regions 5 and 6 . In this configuration, the shock waves associated with the second ramp cause a stronger impinging jet and amplify the heat transfer along the sidewall.

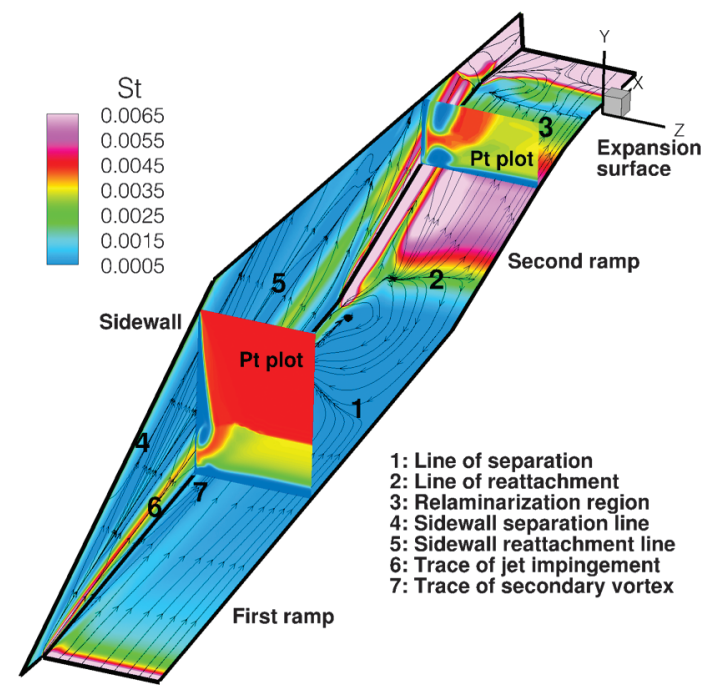

a) CFD (half width)

\section{B. Effects of Sidewall Compression}

Here, the inlet with sidewall compression is considered. The top plot in Fig. 8 illustrates the computed wall pressure along the bottom wall in the symmetry plane. Similar to the configuration without the sidewall compression, the flow is assumed to be laminar on the first ramp. The numerical results are in good agreement with the measurements and the assumption seems to be acceptable. As expected, increasing the sidewall compression increases the pressure in the inlet. The earlier rise of the pressure on the first ramp at larger sidewall compression indicates that the flow separation at the kink between the first ramp and the second ramp is more severe. This is not surprising because, when the sidewall compression is present, the stream tube is compressed by both the ramps and the sidewall convergence, the streamwise pressure gradient is stronger, and the laminar boundary layer separates earlier on the first ramp. However, the measurements were not sufficient to conclude that the size of the separation bubble was predicted quantitatively accurately in the computations.

In the following, the flow features of the $70 \mathrm{~mm}$ insert are discussed because the changes in this case are most pronounced. The flow features in the symmetry plane of the inlet with the $70 \mathrm{~mm}$ insert are shown in the middle plot of Fig. 8 . The background is the Mach contours and the solid lines are the isopressure lines. Consistent with the preceding discussion, the separation bubble in between the ramps

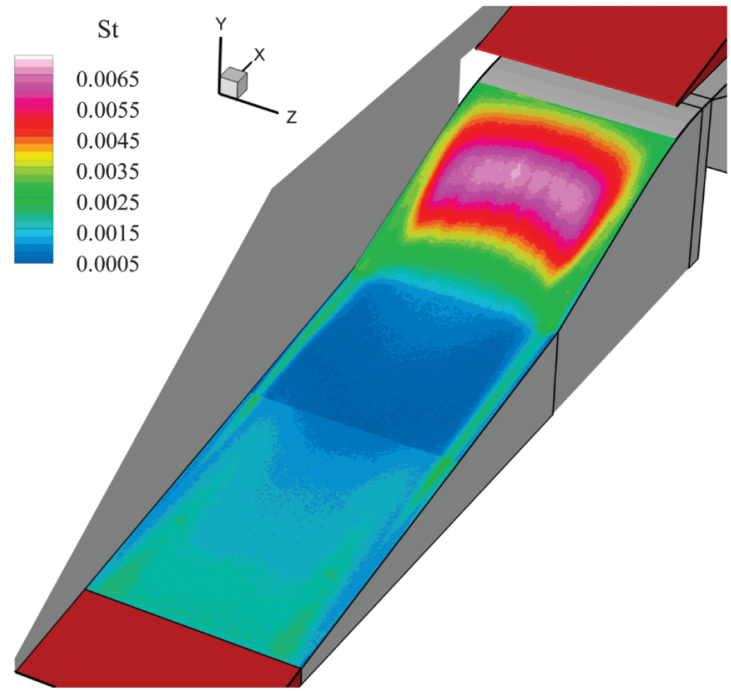

b) Infrared picture (full width)

Fig. 7 Heat transfer contours and stream traces on the surfaces of the inlet without SWC. 

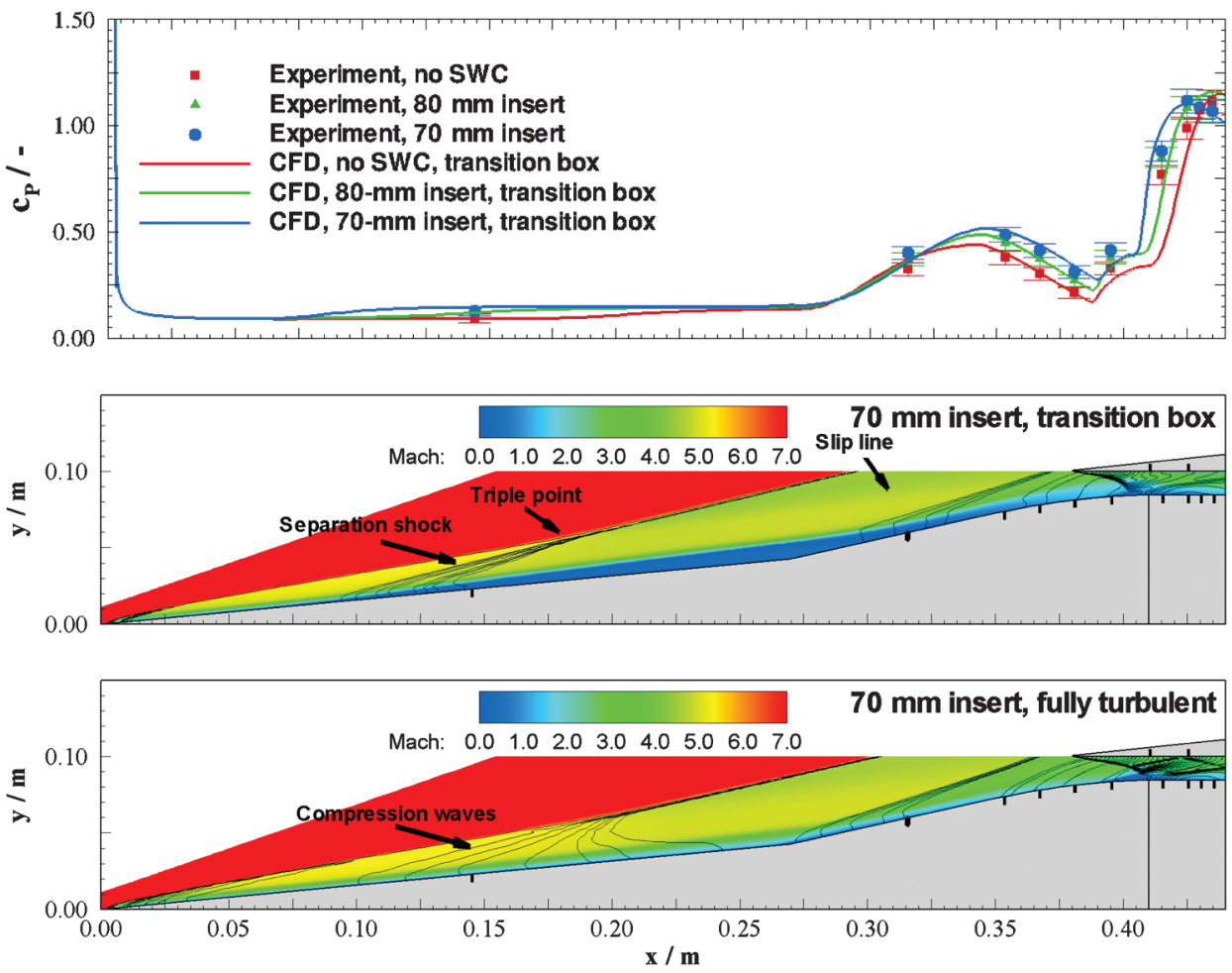

Fig. 8 Pressure coefficient and flow features in the symmetry plane.

is much larger in comparison to the inlet without the sidewall compression (see Fig. 5). This causes the separation shock to move significantly upstream and eventually impinge on the leading-edge shock. The interaction results in an oblique shock wave that is stronger than the original leading-edge shock and a slip line downstream of the triple point (see Fig. 8). Here, the slip line is visible through the moderate change of the Mach contour and the interaction is classified as a type VI Edney's shock-shock interference [22]. To assess the influence of the separation bubble, the flow features of an additional fully turbulent computation are illustrated in the bottom plot of Fig. 8. Because there is no flow separation in this case, the additional compression is achieved more gradually through a series of compression waves on the first ramp and the angle of the final oblique shock wave is smaller than that in the transition box computation, as evident in the figure.

It is now interesting to examine how the presence of sidewall compression modifies the flow structures and imposes more compression in the symmetry plane. Figure 9 shows the flowfield in planes normal to the freestream direction at three different streamwise locations. For the case with the sidewall compression, the results from the fully turbulent computations are also included to isolate the effects of the separation bubble. At the first station $(x=0.07 \mathrm{~m})$, in the configuration without the sidewall compression, the ramp shock is slightly curved upward in the vicinity of the triple point. This curve is due to the requirement that pressure must be equal across the slip line. Here, the ramp shock is equivalent to a "Mach stem" in two-dimensional Mach reflection [23]. With the sidewall compression, the flow must be compressed more in the region close to the sidewall and this is achieved by a larger curve, which extends further toward the symmetry plane. This curve is analogous to a "Mach disk" in a typical symmetric corner flow [24]. The streamwise flow (normal to the $y-z$ plane) below this curve is deflected by an angle larger than the ramp angle and the increase of pressure across the shock wave is larger. Furthermore, the primary vortex is flattened and the associated flow structures are more confined to the sidewall region. This station is upstream of the laminar separation, and so the flowfields in the transition box computation and fully turbulent computation are almost identical.

At the next station $(x=0.21 \mathrm{~m})$, the effect of sidewall compression already extends to the symmetry plane. The ramp shock is being curved more significantly, in comparison with the configuration without SWC, which allows for a greater compression, as explained before. Along the streamwise direction, this must be accompanied by a series of compression waves on the ramp, as seen in the bottom plot of Fig. 8. In case of a laminar flow, the pressure increase due to the compression causes the boundary layer to separate
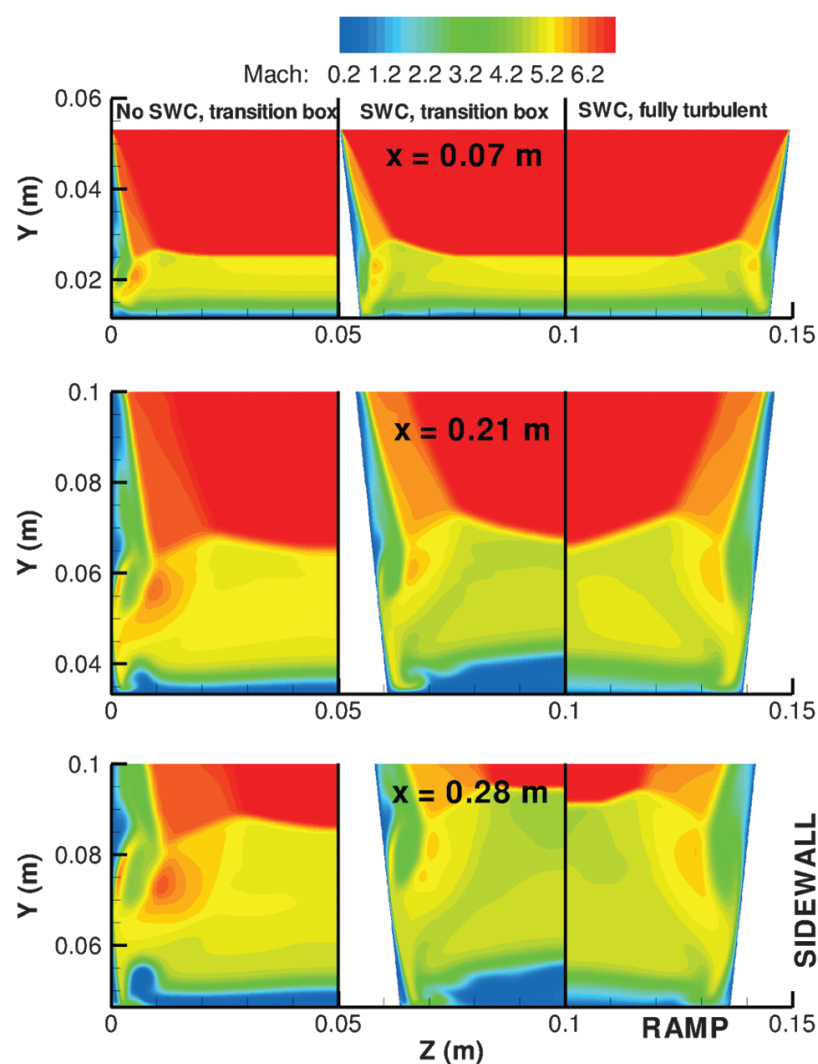

Fig. 9 Mach contours in planes normal to the freestream direction. SWC refers to the inlet with $70 \mathrm{~mm}$ insert. 


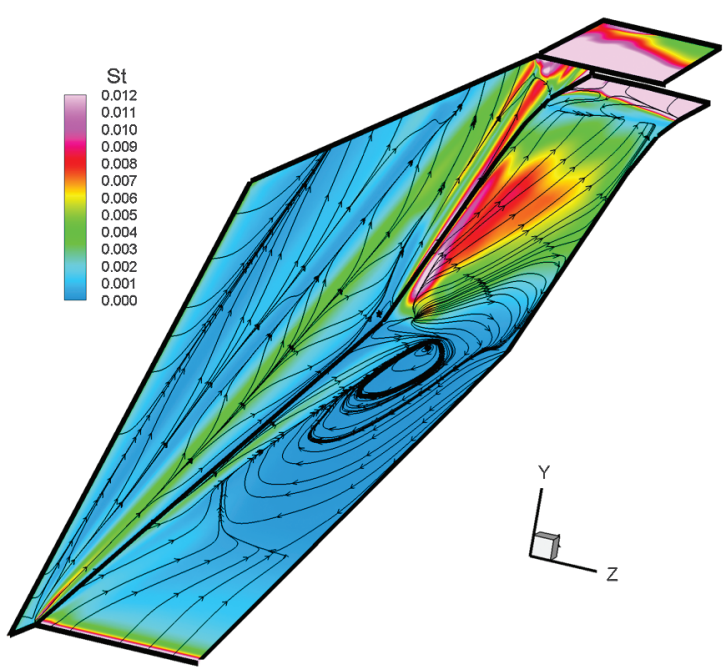

a) CFD (half width)

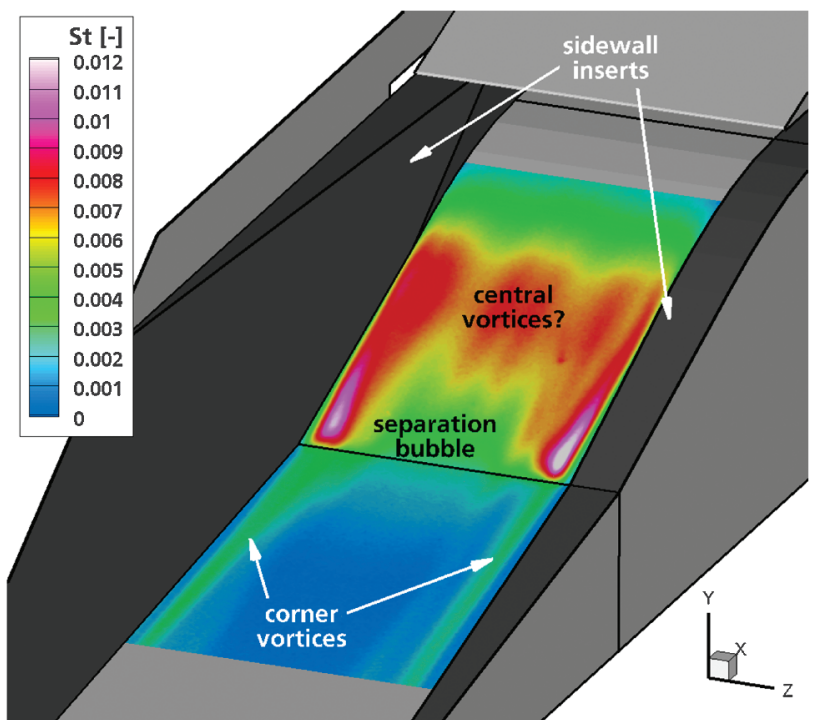

b) Infrared picture (full width)

Fig. 10 Heat transfer contours and stream traces on the surfaces of the inlet with $70 \mathrm{~mm}$ insert.

earlier and results in a larger separation bubble. The increased bubble, in return, causes a strong separation shock to deflect the flow around the separation streamline, which unites with the ramp shock and thus bends it even more upward. This phenomenon can be seen by comparing the ramp shock in the transition box computation to that in the fully turbulent computation. The Mach contour at $x=0.28 \mathrm{~m}$, which is just downstream of the ramp kink, emphasizes the effect even more. For the computation with the presence of the flow separation, the ramp shock is already about to leave the computational domain, leading to a largely spillage drag and more flow losses.

The flow structures on the surface of the inlet with $70 \mathrm{~mm}$ insert are illustrated in Fig. 10. In comparison with Fig. 7a without the sidewall compression, the separation bubble is significantly larger and the heated area on the second ramp is much less uniformly distributed in the spanwise direction. Near the sidewall, a peak heating rate occurs due to the jet impingement of the swept shock-boundary-layer interaction. Reasonable agreement with the measurements from an infrared camera in Fig. 10b can be observed with two small differences: the computed heat transfer at the corner between the first and the second ramps is smaller than the measurement, and the region of high-heat transfer at the end of the second ramp in the infrared picture is missing in the simulation. These discrepancies are attributed to the fact that the RANS calculation is not able to predict separation-induced laminar-turbulent transition accurately. It is believed that the high-heat transfer region at the end of the second ramp is due to the presence of three-dimensional vortex structures [3] , which are not captured in the simulation.

The inlet performance without and with the sidewall compression is summarized in Table 2. The mass flow capture is defined as the ratio between the mass flow rate inside the interior part and the available mass flow rate from the freestream. A good agreement between numerical results and experimental data can be observed. The pressure ratio is defined as the ratio of the pressure at $x=$ $440 \mathrm{~mm}$ (interface between inlet and isolator) and the freestream pressure. It can be seen that, although the static pressure is increased with the larger degree of sidewall compression, both the mass flow capture and the total pressure ratio are reduced. This is because the shock system has a tendency to deflect the flow upward and there are more pressure losses due to the stronger shock waves and the larger separation bubble.

\section{Effects of Relaminarization}

As mentioned previously, to assess the effects of rapid expansion and relaminarization, experiments at two different Reynolds numbers were performed in which the cowl was removed from the inlet. This is to ensure that the flow downstream of the expansion surface can be studied without any disturbances. The two bottom plots in Fig. 11 illustrate the general flowfield, whereas the top plots compare the surface distributions in terms of the pressure coefficient and the Stanton number. $R e_{\infty, m}$ is denoted as "Re" in this figure. In the absence of the cowl, the flow downstream of the second ramp gradually expands and is accelerated across the shoulder. Because the density drops more quickly than the increase of velocity, the boundary layer becomes much thicker downstream of the interaction. Both the wall pressure and the heat transfer are decreased dramatically along the expansion surface. The predicted wall pressure in the symmetry plane is in good agreement with the measurements, whereas the peak heating rate upstream of the expansion is generally overpredicted. This is due to two reasons. First, the actual wall temperature did not remain constant during the experiment. Although the assumption of constant wall temperature may seem to be valid due to short run time (approximately $20 \mathrm{~s}$ ), there are certain hot spots, such as the reattachment point on the second ramp, at which the wall temperature rose significantly during the test. However, the wall temperature is fixed at $300 \mathrm{~K}$ in the computation. Second, RANS turbulence models are not yet at the state where the heat transfer across a flow separation can be predicted with great accuracy. These reasons explain the discrepancy at the peak heating rate. Downstream of this peak, on the expansion surface, the decrease of the Stanton number is predicted reasonably accurately. Here, the upstream Mach number is approximately four, therefore, it can be concluded that RANS models can predict the pressure and heat transfer in a relaminarizing flow at an upstream Mach number larger

Table 2 Inlet performance without and with sidewall compression

\begin{tabular}{lccc}
\hline \hline & No SWC & $80 \mathrm{~mm}$ insert & $70 \mathrm{~mm}$ insert \\
\hline Mass flow capture, experiment, $\%$ & $73 \div 74 \%$ & $60 \div 62 \%$ & $46 \div 54 \%$ \\
Mass flow capture, CFD, \% & $74 \%$ & $63 \%$ & $56 \%$ \\
Area-averaged static pressure ratio, CFD & 25.4 & 28.3 & 30.0 \\
Mass-averaged total pressure ratio, CFD & 0.244 & 0.244 & 0.237 \\
\hline \hline
\end{tabular}



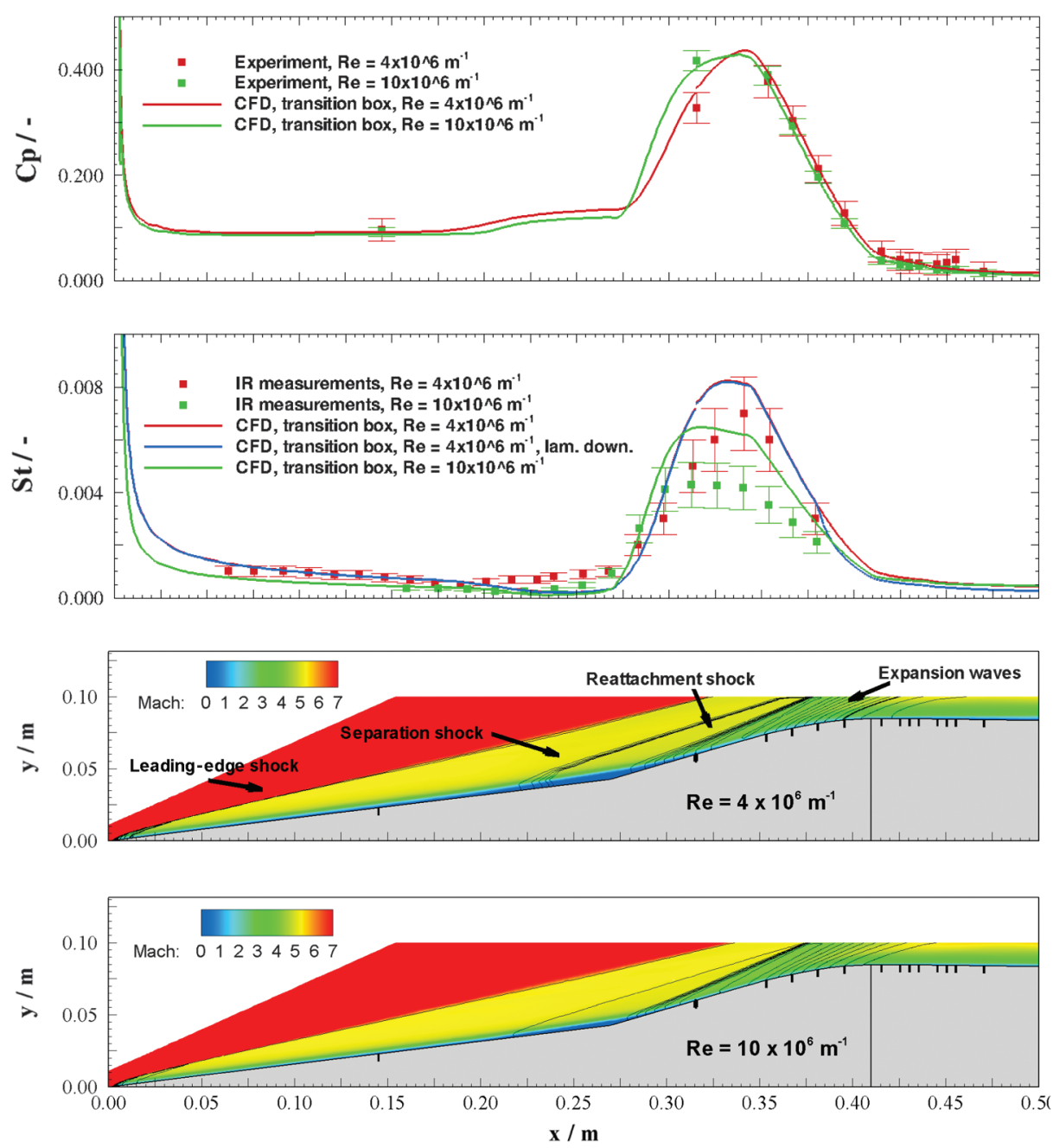

Fig. 11 Pressure and heat transfer in the symmetry plane of the inlet with the cowl removed.

than three (see Nguyen et al. [9,10] for cases at Mach numbers smaller than three).

Figure 11 also illustrates that increasing the Reynolds number has several effects on the flow. These effects are summarized in Table 3 . At the higher Reynolds number, the boundary-layer thickness is smaller on the first ramp and this causes a slightly weaker leadingedge shock. In this context, the boundary-layer thickness is defined as the distance from the wall to a point where the flow velocity is $99 \%$ of the freestream velocity. The Stanton number on the first ramp is also smaller because the freestream density, the freestream velocity, and the total temperature were increased to achieve a higher Reynolds number [see Eq. (2) for the definition of Stanton number]. Because the boundary layer is thinner and the freestream velocity is larger, the flow in the near-wall region has relatively higher momentum to resist the adverse pressure gradient due to the flow turning in between the first and second ramps. Thus, the onset of the laminar separation on the first ramp is delayed and the reattachment on the second ramp is earlier, as indicated in the wall pressure and the contour plots (Fig. 11). This is consistent with the flow features observed in the infrared picture by Häberle and Gülhan [2]. Downstream of the reattachment, the Stanton number distribution forms a plateau at which the peak heating rate remains constant and its value is considerably lower than that at lower Reynolds number (see the Stanton number downstream of the reattachment shock wave in the second plot in Fig. 11). At both Reynolds numbers, the Stanton numbers drop to a similar asymptotic value from the peak value downstream of the expansion surface. The overall decrease in Stanton number across the expansion region is smaller at higher Reynolds number.

The phenomenon of relaminarization will now be analyzed more closely. An additional computation in which the boundary layer is assumed to be fully laminar downstream of the expansion surface $(x>0.38 \mathrm{~m})$ was performed and the results are included in Fig. 11 (labeled as "lam. down"). This helps to quantify how low the Stanton number would be if the flow was fully relaminarized. The heat transfer in this special case is slightly smaller than that in the standard computation, which indicates that the actual flow is close to fully laminar. Figure 12 shows three different velocity profiles plotted in

Table 3 Summary of important flow properties observed in the numerical results in the symmetry plane

\begin{tabular}{cccccc}
\hline \hline Condition & $R e_{\infty, m}, 1 / \mathrm{m}$ & $\delta_{\mathrm{BL}},{ }^{\mathrm{a}} \mathrm{mm}$ & $x_{\text {sep. }}{ }^{\mathrm{b}} \mathrm{m}$ & $x_{\text {reat. }},{ }^{\mathrm{c}} \mathrm{m}$ & $S t$ drop \\
\hline $\mathrm{A}$ & $4 \times 10^{6}$ & 7.86 & 0.204 & 0.296 & 0.0077 \\
$\mathrm{~B}$ & $10 \times 10^{6}$ & 6.60 & 0.212 & 0.285 & 0.0060 \\
\hline \hline
\end{tabular}

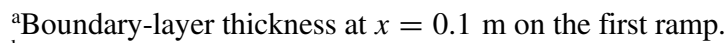

${ }^{b}$ Locations of flow separation in the symmetry plane.

cLocations of reattachment in the symmetry plane.

${ }^{\mathrm{d}}$ Total reduction of Stanton number across the expansion region. 


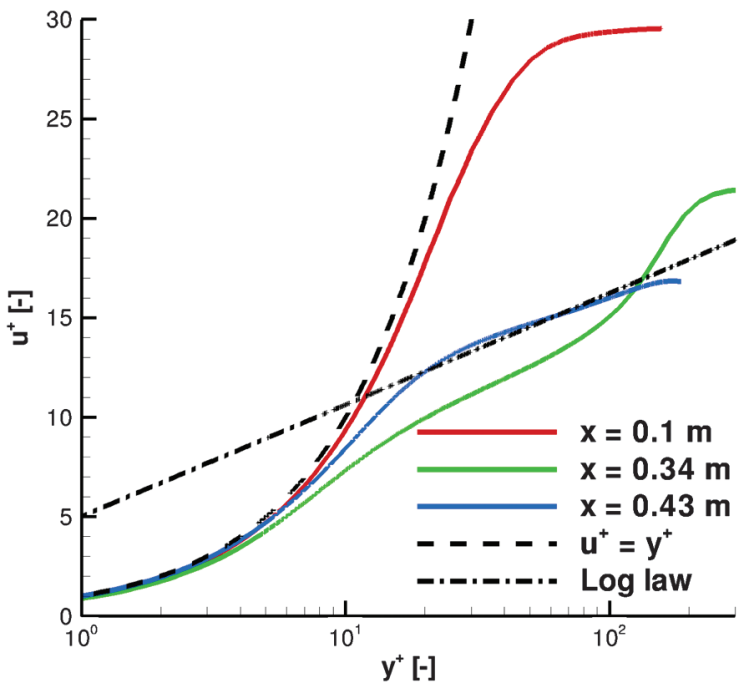

Fig. 12 The van Driest transformed velocity profiles plotted in wall coordinates.

terms of $u^{+}$and $y^{+}$. Each profile is extracted normal to the local wall surface. The first profile $(x=0.1 \mathrm{~m})$ was taken on the first ramp, where the boundary layer is laminar and can be described by the viscous sublayer relationship: $u^{+}=y^{+}$. The second profile $(x=0.34 \mathrm{~m})$ is downstream of the flow reattachment, where the boundary layer is turbulent. Here, the log layer undershoots the standard log law because the boundary layer is still subjected to the adverse pressure gradient. The third profile $(x=0.43 \mathrm{~m})$ represents the relaminarized boundary layer downstream of the expansion

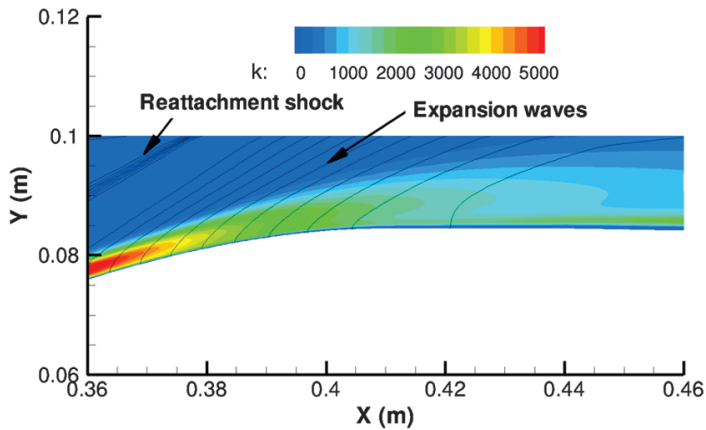

a)

Fig. 13 Effects of rapid expansion on a) turbulent kinetic energy and b) viscosity ratio.

b) region. It can be seen that this profile departs from the log law in which the laminar viscous sublayer is extended, the buffer layer slightly overshoots the log line, and the wake layer collapses. The overall response is very similar to that in the successive distortion experiments performed by Smith and Smits [25] and computations by Nguyen et al. [10].

The contours of turbulent kinetic energy and viscosity ratio in the symmetry plane of the inlet with the cowl removed are plotted in Fig. 13. The solid lines are the isopressure lines and the dashed lines are the former flow features when the cowl was installed. Figure 13a illustrates that the turbulent kinetic energy $k$ is decreased significantly across the expansion waves and further downstream. This reduction is a combined effect of pressure gradient, streamline curvatures, and bulk dilatation (change of mean density). It was shown experimentally [5] and numerically $[9,10]$ that bulk dilatation is the most dominant factor and is more dominant when the expansion is stronger. To further illustrate the extension of the laminar viscous sublayer, Fig. $13 \mathrm{~b}$ shows the ratio of turbulent and dynamic viscosity. Here, the turbulent viscosity is defined as $\mu_{t}=k / \omega$, where $\omega$ is the specific dissipation rate. Upstream of the expansion region, it can be seen that the turbulent viscosity is much larger than the dynamic one, except in the vicinity of the solid wall. Because of both the suppression of turbulence and the expansion of the boundary layer, the region in which the dynamic viscosity is important is enlarged significantly downstream of the inlet shoulder. This observation supports the proposal of the formation of a new laminar sublayer by Narasimha and Sreenivasan [26]. Because the laminar boundary layer is more susceptible to flow separation, this thick laminar sublayer would be partially responsible for the large separation bubble at the entrance of the interior part if the cowl was not removed.

The three-dimensional flow features, in terms of the heat transfer contours and the surface stream traces, are shown in Fig. 14. As

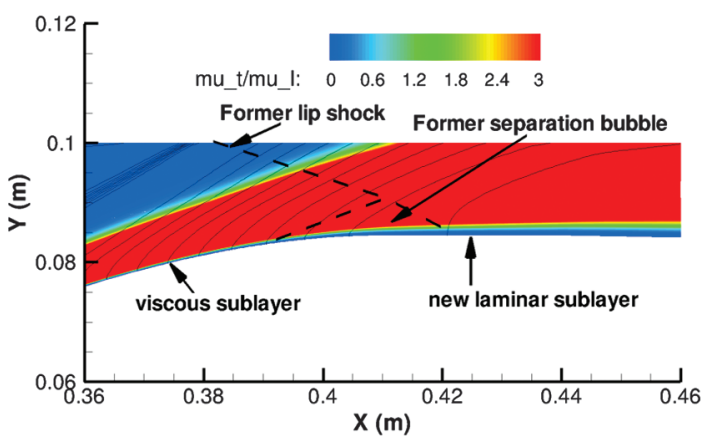

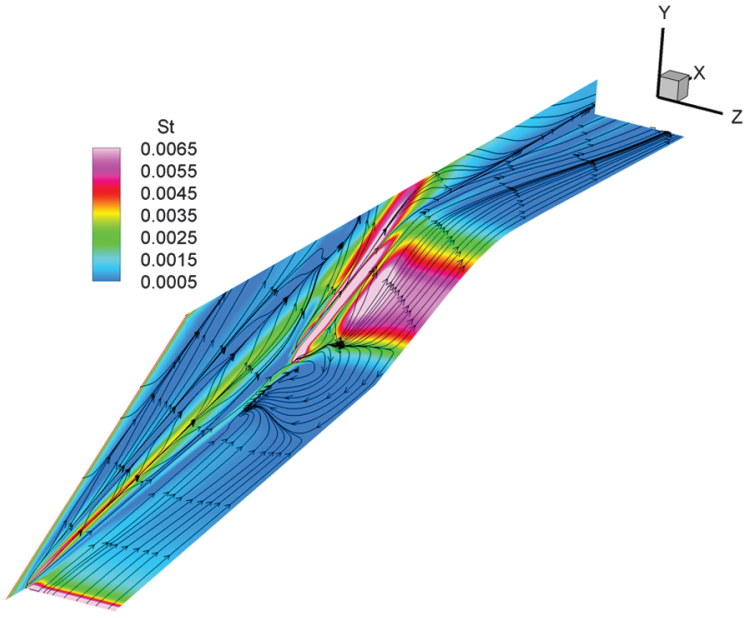

a) Condition DLR A, $\operatorname{Re}_{\infty, m}=4 \times 10^{6} \mathrm{~m}^{-1}$

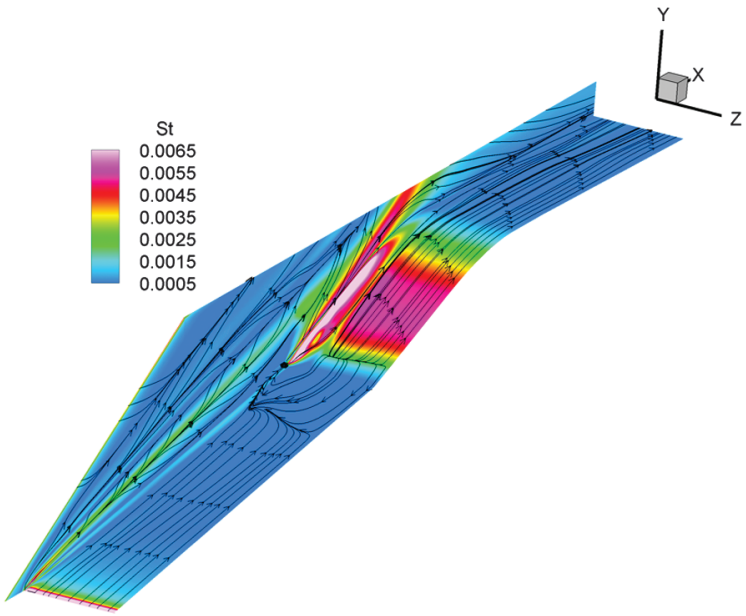

b) Condition DLR B, $\operatorname{Re}_{\infty, m}=4 \times 10^{6} \mathrm{~m}^{-1}$

Fig. 14 Heat transfer contours and surface stream traces on the ramp and the sidewall of the inlet with the cowl removed. 
Table 4 Application of the relaminarization criterion from Narasimha and Viswanath [8] to the flow across the convex shoulder in the scramjet inlet

\begin{tabular}{cccc}
\hline \hline Condition & $M_{\text {upstream }}$ & $R e_{\infty, m}, 1 / \mathrm{m}$ & $\Delta P / \tau_{0}$ \\
\hline $\mathrm{A}$ & 4.0 & $4 \times 10^{6}$ & 47.8 \\
$\mathrm{~B}$ & 4.0 & $10 \times 10^{6}$ & 59.4 \\
\hline \hline
\end{tabular}

discussed before, it can be seen that the flow separation in between the first and the second ramps is smaller at higher Reynolds number. Furthermore, the stream traces are mostly parallel across the expansion region, indicating that relaminarization is two-dimensional. Downstream of the convex surface, a strong streamline divergence toward the symmetry plane is observed, which is probably due to the expansion of the corner vortex that forces the impinging flow away from the sidewall. This effect is less severe at higher Reynolds number.

\section{Criterion of Relaminarization}

The criterion of relaminarization from Narasimha and Viswanath [8] mentioned in Sec. I can be used to assess the occurrence of relaminarization. However, it was derived based on a collection of experiments where the upstream Mach number is less than three and it is only valid in this regime. Because the Mach number upstream of the expansion region in the scramjet inlet considered here is approximately four, the results of this work provide a chance to test the possibility to extend the criterion to higher Mach numbers. The computed criteria from the numerical results are given in Table 4 . It can be seen that, at both Reynolds numbers, the ratio $\Delta P / \overline{\tau_{0}}$ is smaller than 70 , even though the boundary layer is very close to fully laminar as discussed previously. This is because the velocity gradient at the wall is much larger when the freestream Mach number is larger, thus $\tau_{0}$ is increased significantly and causes a smaller value of $\Delta P / \tau_{0}$ despite a strong reduction in pressure. Therefore, it seems that a Mach number correction is required to extend the use of the criterion beyond Mach 3 . This correction cannot be obtained here due to the limited available data at higher Mach numbers, but it is an interesting topic for future work.

\section{Conclusions}

Computations were carried out to assess the effects of sidewall compression and relaminarization on the performance of a scramjet inlet. Sidewall compression was achieved by introducing a smooth contraction along the exterior compression of the inlet. Good agreement with the wall pressure measurements was observed. The flow features suggest that a convergent sidewall compresses the flow by curving the ramp shock upward in the vicinity of the sidewall and extending this curve toward the symmetry plane. It was also demonstrated that this phenomenon would be enhanced if the flow separated on the ramp. The numerical results indicate that the weak sidewall compression alters the inlet performance significantly. Although the static pressure is increased due to more compression, both the mass flow capture and the total pressure ratio are reduced. This is because the shock system has a tendency to deflect the flow upward and there are increased pressure losses due to stronger shock waves and larger separations.

Relaminarization was studied experimentally and numerically by testing the inlet with the cowl removed at two different Reynolds numbers. The computations predict the measured wall pressure accurately. The predicted heat transfer is acceptable except around the reattachment point on the second ramp, where the peak heating rate is overpredicted. This is because the wall temperature increased significantly at this point in the experiment and this was not taken into account in the computations. Another possible reason is the limitation of the Reynolds-averaged Navier-Stokes turbulence model in predicting flow separation. Downstream of the expansion surface, both the Stanton number and the turbulent kinetic energy are reduced dramatically. The flow is found to be quasi laminar and there is a formation of a thick viscous sublayer. At higher Reynolds number, the overall reduction of heat transfer is smaller, indicating that the flow is less relaminarized.

Several conclusions can be drawn from this work that are useful in designing a scramjet inlet. First of all, inlet designers should beware that sidewall compression causes the ramp shock to bend upward and, if the location of the cowl lip is unchanged, more mass flow spillage will occur. Second, due to the higher pressure gradient, the boundary layer inside the inlet will be more likely to separate and result in a significant reduction of performance. This may be avoided if the boundary layer is turbulent, as illustrated in the preceding section. Finally, because relaminarization causes the formation of a thick laminar sublayer, the boundary layer is prone to a strong separation under adverse pressure gradient due to shock wave boundary-layer interaction. Thus, it is probably a good idea to impose the shock wave upstream of the expansion region, where the boundary layer is still turbulent, so that the probability of a massive flow separation is reduced.

\section{Acknowledgments}

This work was supported by the German Research Foundation (DFG) within the framework of the GRK 1095 "AeroThermodynamic Design of a Scramjet Propulsion System for Future Space Transportation Systems" and the GSC 111 Aachen Institute for Advanced Study in Computational Engineering Science (RWTH Aachen University). Computing resources were provided by the RWTH Aachen University Center for Computing and Communication and the Forschungszentrum Jülich.

\section{References}

[1] Gaisbauer, U., Weigand, B., and Reinartz, B., "Research Training Group GRK 1095/1: Aero-Thermodynamic Design of a Scramjet Propulsion System," Proceedings of 18th ISABE Conference, International Symposium of Air-Breathing Engines, AIAA Paper ISABE-2007-1131, Beijing, China, 2007.

[2] Häberle, J., and Gülhan, A., "Investigation of Two-Dimensional Scramjet Inlet Flowfield at Mach 7," Journal of Propulsion and Power, Vol. 24, No. 3, 2007, pp. 446-459. doi: $10.2514 / 1.33545$

[3] Hohn, O., and Gülhan, A., "Experimental Investigation on the Influence of Sidewall Compression on the Flowfield of a Scramjet Inlet at Mach 7," AIAA Paper 2011-2350, 2011.

[4] Holland, S. D., "Computational Parametric Study of SidewallCompression Scramjet Inlet Performance at Mach 10," NASA TM4411, Feb. 1993.

[5] Dussauge, J.-P., and Gaviglio, J., "The Rapid Expansion of a Supersonic Turbulent Flow: Role of Bulk Dilatation," Journal of Fluid Mechanics, Vol. 174, Jan. 1987, pp. 81-112. doi:10.1017/S0022112087000053

[6] Smith, D. R., and Smits, A. J., "The Rapid Expansion of a Turbulent Boundary Layer in a Supersonic Flow," Theoretical and Computational Fluid Dynamics, Vol. 2, Nos. 5-6, 1991, pp. 319-328. doi:10.1007/BF00271471

[7] Sreenivasan, K. R., "Laminarescent, Relaminarizing and Retransitional Flows," Acta Mechanica, Vol. 44, Nos. 1-2, 1982, pp. 1-48. doi:10.1007/BF01190916

[8] Narasimha, R., and Viswanath, P. R., "Reverse Transition at an Expansion Corner in Supersonic Flow," AIAA Journal, Vol. 13, No. 5 , 1975, pp. 693-695. doi: $10.2514 / 3.49793$

[9] Nguyen, T., Behr, M., and Reinartz, B., "Numerical Investigation of Compressible Turbulent Boundary Layer over Expansion Corner," AIAA Paper 2009-7371, 2009.

[10] Nguyen, T., Vukovic, M., Behr, M., and Reinartz, B., "Numerical Simulations of Successive Distortions in Supersonic Turbulent Flows," AIAA Journal, Vol. 50, No. 11, 2012, pp. 2365-2375. doi:10.2514/1.J051465

[11] Henckels, A., and Gruhn, P., "Study on Aerothermal Effects of Viscous Shock Interaction in Hypersonic Inlets," Proceedings of 5th European Symposium on Aerothermodynamics for Space Vehicles, ESA Publication Division, Cologne, Germany, 2005.

[12] Bramkamp, F. D., Lamby, P., and Müller, S., "An Adaptive Multiscale Finite Volume Solver for Unsteady and Steady Flow Computations," 
Journal of Computational Physics, Vol. 197, No. 2, 2004, pp. 460-490. doi:10.1016/j.jcp.2003.12.005

[13] Wada, Y., and Liou, M. S., "An Accurate and Robust Flux Splitting Scheme for Shock and Contact Discontinuities," SIAM Journal on Scientific Computing, Vol. 18, No. 3, 1997, pp. 633-648. doi: $10.1137 / \mathrm{S} 1064827595287626$

[14] Krause, M., "Numerical Analysis of Transition Effects for Scramjet Intake Flows," Ph.D. Thesis, RWTH Aachen University, Aachen, Germany, 2010.

[15] Menter, F. R., "Two-Equation Eddy-Viscosity Turbulence Models for Engineering Applications," AIAA Journal, Vol. 32, No. 8, 1994, pp. $1598-1605$. doi: $10.2514 / 3.12149$

[16] Brodersen, O., Ronzheimer, A., Ziegler, R., Kunert, T., Wild, J., and Hepperle, M., "Aerodynamic Applications using MegaCads," Proceedings of the 6th International Conference on Numerical Grid Generation in Computational Field Simulations, edited by Cross, M., Haeuser, J., Soni, B. K., and Thompson, J. F., International Society of Grid Generation, Mississippi, 1998, pp. 793-802.

[17] Alvi, F. S., and Settles, G. S., "Physical Model of the Swept Shock Wave/Boundary-Layer Interaction Flowfield," AIAA Journal, Vol. 30, No. 9, 1992, pp. 2252-2258. doi: $10.2514 / 3.11212$

[18] Smits, A. J., and Dussauge, J.-P., Turbulent Shear Layers in Supersonic Flow, Springer, New York, 2006, pp. 356-360.

[19] Kubota, H., and Stollery, J., "An Experimental Study of the Interaction Between a Glancing Shock Wave and a Turbulent Boundary Layer," Journal of Fluid Mechanics, Vol. 116, March 1982, pp. 431-458. doi:10.1017/S0022112082000548
[20] Knight, D. D., Badekas, D., Horstman, C. C., and Settles, G. S., "Quasiconical Flowfield Structure of a Three-Dimensional Single Fin Interaction," AIAA Journal, Vol. 30, No. 12, 1992, pp. 2809-2816. doi: $10.2514 / 3.48972$

[21] Knight, D. D., Horstman, C. C., Shapey, B., and Bogdonoff, S., "Structure of Supersonic Turbulent Flow Past a Sharp Fin," AIAA Journal, Vol. 25, No. 10, 1987, pp. 1331-1337. doi: $10.2514 / 3.9787$

[22] Babinsky, H., and Harvey, J. K., Shock Wave-Boundary-Layer Interactions, Cambridge Univ. Press, Ithica, NY, 2011, pp. 11-16.

[23] Lu, F. K., "Quasiconical Free Interaction Between a Swept Shock and a Turbulent Boundary Layer," AIAA Journal, Vol. 31, No. 4, 1993, pp. 686-692. doi: $10.2514 / 3.11604$

[24] D'Ambrosio, D., and Marsilio, R., "Shock-Induced Separated Structures in Symmetric Corner Flows," NASA CR-198239, Dec. 1995

[25] Smith, D. R., and Smits, A. J., "The Effects of Successive Distortions on a Turbulent Boundary Layer in a Supersonic Flow," Journal of Fluid Mechanics, Vol. 351, Nov. 1997, pp. 253-288. doi:10.1017/S0022112097006988

[26] Narasimha, R., and Sreenivasan, K. R., "Relaminarization in Fluid Flows," Advances in Applied Mechanics, Vol. 19, 1979, pp. 221-309. doi:10.1016/S0065-2156(08)70311-9

R. Bowersox Associate Editor 